\title{
El aporte de Samuel Pinheiro Guimarães al acervo eidético de los Estudios Internacionales
}

\author{
Andrés Figueroa Jiménez ${ }^{1}$. \\ “-Tenemos una idea permanente - Hay bloques. Habiendo bloques, esos bloques se \\ disputan el espacio y el proceso de globalización internacional que es un proceso económico y que \\ tiene también aspectos políticos en la reglamentación de los países en torno a ciertas políticas". \\ Samuel P. Guimarães, (2011). “Los caminos de la integración ¿Hacia dónde vamos?”.
}

\section{Resumen:}

Este escrito expone la contribución teorética de Samuel Pinheiro Guimarães al acervo eidético de la multidisciplina Estudios Internacionales. Para un ejercicio adecuado de aquello se desarrollan los siguientes contenidos: Primero, se define a los Estudios Internacionales como un campo dedicado a realizar exámenes sobre el poder a partir de una arquitectura específica de dimensiones y a través de variados niveles y escalas. Segundo, se enuncia sobre el poder, en tanto que objeto de análisis en los Estudios Internacionales, como un concepto performativo que se define por entregar una resolución de la vinculación entre ideas, teorías y prácticas en el campo de la política. Tercero, se presentan y declaran a los Estudios Eidéticos como un área disciplinar funcional al estudio del poder como concepto performativo y como núcleo analítico de los Estudios Internacionales desde el posibilitar indagaciones sobre las ideas políticas a partir del empleo de la categoría "acervo eidético". Cuarto, se presenta la biografía profesional y bibliografía intelectual de Pinheiro Guimarães en los primeros años del siglo XXI considerando en detalle a estos elementos como un arsenal de instrumentos teoréticos que fortalecen a los Estudios Internacionales y sus labores de producción de conocimiento en sentido básico y aplicado.

Palabras Clave: Acervo eidético, Capacidad performativa de las ideas, Estudios Eidéticos, Estudios Internacionales, Samuel Pinheiro Guimarães.

\footnotetext{
${ }^{1}$ Chileno. Magíster en Estudios Internacionales por el Instituto de Estudios Avanzados (IDEA) de la
} Universidad de Santiago de Chile. Email: andres.figueroa@usach.cl / ORCID ID: 0000-0003-3471-4303. 


\section{Introducción}

\section{Multidisciplina Estudios Internacionales en sentido básico y aplicado:}

Los Estudios Internacionales son un campo de producción de conocimiento, indagaciones y análisis sobre problemáticas ligadas al poder en múltiples niveles y escalas perteneciente a las Ciencias Sociales y Humanidades con un carácter multidisciplinar. Aquí, se asume a los Estudios Internacionales como multidisciplina, porque sus tareas se abocan, en un sentido básico, al desarrollo de exámenes (comprensiones y explicaciones) sobre las ideas, las acciones y los vínculos de actores nacionales y subnacionales, estatales y no estatales, formales e informales, legales e ilegales cuyas actividades tienen la capacidad de generar influencia - desde recursos de poder - sobre las percepciones, las políticas y sobre el curso o dirección de las relaciones entre dos o más agentes internacionales (sean Estados o actores no estatales) ${ }^{2}$ (Muñoz, 1980; Orrego Vicuña, 1981; Bello, 2013; Caro y Rodríguez, 2013; Rodríguez, 2013; Ross, 2020).

Las dimensiones mentadas de los Estudios Internacionales poseen un carácter eidético, político, económico, social, cultural y estratégico (Devés y Álvarez, 2020; Ross, 2020). Así, su estudio y análisis impone el uso de diferentes enfoques teóricos, atendiendo a la naturaleza especifica de las unidades de análisis. Los Estudios Internacionales, así definidos, suponen el empleo de un conjunto de métodos de pesquisa cualitativos y cuantitativos, a los cuales los investigadores pueden recurrir de una manera convencional o de forma heterodoxa innovando en su combinación en función de unidades de análisis y objetivos de estudio (Muñoz, 1980; Orrego Vicuña, 1981; Navarrete, Morales y Figueroa, 2005; Caro y Rodríguez, 2013; Ross, 2020) ${ }^{3}$.

Los Estudios Internacionales, siguiendo una nomenclatura clásica, se encuentran fuertemente pero no exclusivamente, constituidos como un rendimiento o producto teórico, epistemológico y metodológico - analítico del denominado "Tercer Debate de las Relaciones Internacionales" entre Realismo y Transnacionalismo. Aquí, los Estudios Internacionales concentran sus labores de pesquisa sobre relaciones y flujos entre actores y dimensiones de diverso carácter, a saber, personas, bienes e influencias en aristas como la política, economía,

\footnotetext{
${ }^{2}$ En Chile, ejemplos de este entendido de los Estudios Internacionales los constituyen los trabajos realizados en el Instituto de Estudios Internacionales (IEI) de la Universidad de Chile desde 1966 y del Instituto de Estudios Avanzados (IDEA) de la Universidad de Santiago de Chile a partir de 1993. En este último caso, el entendido toma forma a través de la creación del Doctorado en Estudios Americanos (1998), las Jornadas de Estudios Internacionales (1999) y los Magísteres en Política Exterior (2000) y Estudios Internacionales (2003) (Ross, 2020).

${ }^{3}$ El entendido aquí expuesto de los Estudios Internacionales no se trata de un intento ingenuo y estéril de reclamación de autonomía disciplinar. Por el contrario, implica una especificación de dimensiones analíticas fundamentales para dar arquitectura a labores de investigación con cariz teórico o metodológico - técnico y en un sentido básico y aplicado. Se prioriza un entendido sobre los Estudios Internacionales donde estos se caracterizan, como condición necesaria, por una ampliación de la perspectiva para el examen crítico de problemáticas. Esta ampliación va desde lo estrictamente institucional hacia lo social e histórico y constituye una manera de hacer investigación social empírica en el área del poder que persigue distanciarse, al menos en algún grado, de la Ciencia Política, las Ciencias Administrativas y las Relaciones Internacionales obsecuentes al "mainstream" anglosajón y mayoritariamente comprometidas con procedimientos electorales y con una democracia de partidos (Mella, 2012; Bello, 2013; Salomón, 2013; Heiss, 2015; Ross, 2020).
} 
sociedad y estrategia. En consecuencia, en un sentido básico, los Estudios Internacionales son un campo cuya arquitectura obedece a una convergencia relativamente flexible de enfoques, cuyo objetivo es la comprensión de espacios, procesos y temáticas atendiendo tanto a los ejes, discusiones o debates del norte (Ej. EUA o U.K.) así como a los análisis desarrollados desde la Historia, Sociología, Ciencia Política, Cultura y Estudios de Seguridad y Defensa en una orientación multidireccional: sur $\rightarrow$ norte, norte $\rightarrow$ sur y sur - sur (Rodríguez, 2013; Ross, 2020).

En un sentido aplicado, la multidisciplina de los Estudios Internacionales orienta su producción de conocimiento respecto de dimensiones ligadas al poder en múltiples niveles y escalas, al nutrir o fundamentar procesos de análisis, gestión, formulación, implementación y evaluación de políticas y políticas públicas. Lo anterior, siempre develando las vinculaciones o afecciones multinivel o escalares al interior de asuntos o problemáticas (Viacava, 2012; Ross, 2020).

Desde su sentido aplicado, en suma, los Estudios Internacionales son una multidisciplina que se dedica al estudio del poder y a la paralela arquitectura (diseño, implementación y evaluación) de políticas y políticas públicas en tanto que asumidas las mismas como alternativas de actuación o como soluciones para hacer frente a problemas y problemáticas generados por la predominante realidad actual de interdependencia global creciente (Keohane y Nye, 1989; Ross, 2020).

\section{Poder como concepto performativo:}

En el apartado previo, se definió a los Estudios Internacionales como una multidisciplina que examina, comprende y explica problemáticas ligadas al poder posibles de ocurrir en múltiples niveles y escalas. Además, se refirió que el mentado examen, comprensión y explicación, se lleva a cabo a través del empleo de una arquitectura de dimensiones de estudio y análisis a partir de las cuales, en un momento posterior, se generan rendimientos de conocimiento en un sentido básico y aplicado.

Ahora bien, una de las dimensiones de estudio y análisis constituyentes de los Estudios Internacionales son las ideas ${ }^{4}$. Aquí, dado que se adscribe al criterio bajo el cual las ideas constituyen a la vez un recurso y un espacio para la generación de influencia a través del operar como elementos de poder. Lo expuesto, puede comprenderse mejor desde el entender al poder como una idea y/o concepto performativo, en tanto que un lugar, un proceso intelectual y práctico en el que se produce y resuelve la vinculación entre ideas, teorías y prácticas o acciones en el campo de la política (Mella, 2010 y 2012). La enunciación anterior, se fundamenta en los siguientes criterios:

\footnotetext{
${ }^{4}$ Un entendido básico de las Ideas en este texto sigue la noción de J. Mill en su obra: “Análisis de los fenómenos de la mente humana" (1829). Aquí, las ideas son "apariencias" mentales resultantes de la asociación, combinación mental (abstracción) de sensaciones, experiencia (s) sincrónicas o sucesivas ligadas a lo real. Ahora bien, aquí se suscribe a los procesos de ensamblaje complejos, conceptuales/modelares o "tesis/topos" (Colombi, 2006), que cubren variadas escalas y dimensiones de fenómenos e interesan, todavía más, aquellos que pueden estar dotados de un racional (con sentido lógico) impacto o efecto sociopolítico (Freeden, 2013).
} 
Al interior de la Teoría Política contemporánea, existen tres maneras de relacionar las ideas políticas con las prácticas. Primero, considerando que las ideas determinan a las prácticas. Segundo, asumiendo que las prácticas determinan a las ideas o tercero, creyendo que las ideas y prácticas evolucionan por sendas independientes. El primer supuesto significa que, bajo ciertas condiciones, los conceptos políticos debieran influir las prácticas políticas, lo que algunos autores denominan como la "capacidad performativa de las ideas" (Austin, 2008) El segundo supuesto denota que las condiciones del proceso político determinan, bajo ciertos requisitos, el pensamiento político. Entonces, autorías hablan de la "condición o posición enunciativa", el "horizonte hermenéutico", la "economía de los intercambios lingüísticos" y la "fuerza ilocutiva". El tercer supuesto señala que teoría y práctica no se condicionan en ningún caso, lo que constituye la creencia básica del positivismo (Touchard, 2006; Austin, 2008; Mella, 2010 y 2012).

En este documento, se trabaja sobre la base de las dos primeras posibilidades asumidas como principios o ejes orientadores en los Estudios Internacionales. Lo expuesto, bajo el entendido que los conceptos son un reflejo de la realidad $\mathrm{y}$, simultáneamente, contribuyen a determinarla o al menos a orientarla en un sentido específico. Esta condición de las ideas provoca que el pensamiento político constituya una especie de "zona gris", un espacio donde coexisten e interactúan, vía mecanismos y soportes $\left(\mathrm{Ej}\right.$. Documentos $\left.{ }^{6}\right)$ formales o informales, por una parte, el mundo de las prácticas y la experiencia concreta y, por otra parte, la dimensión de percepciones e ideas, la dimensión imaginaria, aspiracional y discursiva de la vida social y sus actores (Touchard, 2006; Auyero, 2007; Mella, 2010 y 2012).

Bajo las referidas premisas, en los apartados siguientes se presentará a la obra, teoría y principales conceptos desarrollados respecto a asuntos y problemáticas internacionales globales por el político y académico brasileño Samuel Pinheiro Guimarães al interior de los primeros años del siglo XXI (1999/2000 - 2005). Aquí, considerando que obras, teoría y

\footnotetext{
5 J. L. Austin desde la Filosofía Analítica del siglo XX en su obra de 1962 "Cómo hacer cosas con palabras", establece su Teoría de la Performatividad en los enunciados lingüísticos. Desde la perspectiva positivista, un enunciado constituye la expresión de una proposición, es decir, de una pieza discursiva cuyo significado puede ser verdadero o falso. Todo enunciado se encuentra dotado de una estructura formal lógica: 1) Sujeto + 2) Verbo + 3) Predicado o si se quiere: 1) Argumento + 2) Predicado (Verbo + Complemento). Contrario a la visión positivista, para Austin el lenguaje y las ideas expresadas a través del mismo no sólo pueden operar como un conjunto de instrumentos descriptivos - constatativos respecto a la realidad, sino que, además, pueden adquirir un carácter realizativo. Para Austin, el lenguaje, las ideas enunciadas no sólo informan la realización de hechos, actos determinados, sino que pueden tener efectos, rendimientos e influencia sobre la realidad. A este tipo de lenguaje Austin denomina como enunciados “performativos" o "lenguaje ejecutivo”. ¿Cómo el lenguaje, las ideas enunciadas pueden tener capacidad performativa? Específicamente al, las ideas, en el proceso que va desde su enunciación inicial y hasta en sus efectos, el poder dotarse de tres fuerzas: 1) Fuerza locutiva (Referida a la frase en si misma), 2) Fuerza ilocutiva (ligada a la intención y significación de las ideas), 3) Fuerza perlocutiva (vinculada a los acontecimientos, conductas y/o acciones sucesivas producidas como efecto de la frase). Para Austin tanto la fuerza ilocutiva como la fuerza perlocutiva otorgan la cualidad de performatividad a los enunciados. Lo anterior, siempre en conformidad con un contexto o conjunto de convenciones que ordena las comprensiones mayores sobre el discurso (Austin, 2008). En suma, en este trabajo se atribuye a las ideas y al lenguaje político que las evidencia, la tipología de fuerzas de Austin como elementos constituyentes.

${ }^{6}$ Libros, Artículos, Testimonios, Reportes, Notas de terreno entre otros soportes. Para Auyero, (2007) estos soportes son operados por figuras que depositan y transmiten en ellos sentidos (significados) y percepciones. Estos sentidos y percepciones pueden ser sometidos a especulación, reflexión y problematización por otros agentes. Aquí, se abre una zona gris capaz de nutrir la comprensión de los hechos y la orientación de conductas.
} 
sobre todo sus conceptos fundamentales, lo que hacen, es alimentar a un acervo de pensamiento latinoamericano en problemáticas internacionales y mundiales (Devés y Álvarez, 2020). Lo anterior, en tanto que por una parte son elementos que han representado parcialmente imágenes de procesos en asuntos internacionales - globales y por otra, también son artefactos que han tenido eficacia performativa para generar, dirigir y sustentar prácticas políticas (Touchard, 2006; Mella, 2010 y 2012).

Ahora bien, antes de presentar la obra, teoría y principales conceptos sobre asuntos internacionales elaborados por Pinheiro Guimarães, se procederá a tratar en la siguiente sección a elementos para entender el ámbito disciplinar Estudios Eidéticos. Aquí, bajo la consideración de que al interior de esta área existen aspectos útiles para pesquisar, indagar y ejecutar rastreos sobre ideas políticas y elementos de pensamiento.

\section{Estudios Eidéticos}

En relación con lo hasta aquí expuesto, en esta sección se referirá sobre los Estudios Eidéticos como una disciplina completo funcional al estudio del poder como concepto performativo y asumiendo al mismo, (al poder), como una imagen, como un indicador de un núcleo analítico o dimensión constitutiva de los Estudios Internacionales, a saber, las ideas. Lo anterior, se desarrolla dado que los Estudios Eidéticos constituyen un área con características que posibilitan el indagar y desarrollar exámenes sobre las ideas políticas a través del uso de herramientas teoréticas como la categoría específica "acervo eidético".

\section{Definición Estudios Eidéticos:}

Siguiendo las consideraciones desarrolladas por Eduardo Devés-Valdés y Andrés Kozel a 2018 en su trabajo denominado "Estudios Eidéticos", aquí se entenderá por Estudios Eidéticos a un espacio multidisciplinar dedicado al desarrollo de conocimientos sobre el pensamiento e ideas, dedicado al examen de la "vida de las ideas" en regiones del mundo a través de variados enfoques ${ }^{7}$. Específicamente, los Estudios Eidéticos se definen por tratar sobre la vida de las ideas en tanto que pesquisar la historia, realidades de las ideas y las perspectivas dedicadas al abordaje del pensamiento e ideas en diversos lugares del mundo. Lo anterior, haciendo uso de conceptos o definiciones, criterios de clasificación, análisis de hipótesis sobre el pensamiento e ideas, el tratamiento de redes intelectuales ${ }^{8}$ y la operación analítica sobre el papel desempeñado por intelectualidades y medioambientes o ecosistemas intelectuales en procesos como la circulación de elementos de pensamiento e ideas.

La principal finalidad de los Estudios Eidéticos al tratar la vida de las ideas, consiste en generar información sobre el pensamiento y las ideas que permita alcanzar un estadio de mayor y mejor pensamiento societal sobre problemas y asumido esto, como una situación de

\footnotetext{
${ }^{7}$ Disciplinas cercanas al estudio de las ideas son: Filosofía, Historia, Estudios Culturales, Ciencias Sociales, Literatura y Lingüística. Como disciplinas más alejadas podemos mencionar a las Ciencias Cognitivas, Biología e Ingeniería (Devés-Valdés y Kozel, 2018).

${ }^{8}$ Para conocer más sobre redes intelectuales como puntos de origen y confluencia de ideas con capacidad de influencia, donde operan aparejos políticos, revistas, congresos, encuentros internacionales, estudios universitarios y lecturas de intelectuales abordados, véase: Devés-Valdés, (2012).
} 
mayor y mejor capacidad para comprender problemáticas y formular soluciones en respuesta a las mismas y sus derivadas (Devés-Valdés y Kozel, 2018; Devés-Valdés, 2019).

¿Cómo específicamente los Estudios Eidéticos examinan la vida de las ideas y posibilitan desde aquello un estadio de mayor y mejor capacidad para el entendimiento de problemáticas y el construir alternativas para su tratamiento? A modo de respuesta, aquí se sostiene que los Estudios Eidéticos lo que hacen es emplear categorías metodológico - analíticas como entidades que permiten la detección de elementos de pensamiento e ideas. Para ejemplificar, se presentará la categoría "acervo eidético".

\section{Categoría9 acervo eidético:}

Al interior de los Estudios Eidéticos, la categoría acervo eidético refiere a una construcción teorética diseñada, a nuestro juicio, para revelar y orientar las explicaciones de elementos clave al interior de entidades eidéticas como el pensamiento de áreas (Ej. Latinoamérica) o el pensamiento desarrollado por una autoría o intelectualidad. Así, los aspectos esenciales del pensamiento e ideas que permite identificar esta categoría a través de su explicitación son los siguientes: Primero, temas y obras. Segundo, ideas centrales. Tercero, conceptos disciplinares fundamentales (Devés-Valdés, 2019). Una estilización o refinación específica del instrumento mentado está presente también en la siguiente arquitectura: 1) Teorías y enfoques teóricos. 2) Escuelas de pensamiento. 3) Conceptos y 4) Doctrinas (Devés-Valdés y Álvarez, 2020). En este documento se prioriza, por su mayor facilidad operativa, la primera formulación.

Los aspectos o dimensiones que dotan de arquitectura a la categoría acervo eidético, a nuestro modo de ver, resultan útiles para la realización de rastreos e indagaciones sobre entidades eidéticas porque nos permiten adentrarnos en cuestiones como las siguientes: 1) Los referentes empíricos específicos de temas e ideas centrales pertenecientes a pensamientos y/o autorías. 2) La estructura y relaciones empíricas, vinculaciones entre fenómenos subyacentes a conceptos particulares. 3) Las uniones o redes complejas de conceptos y generalizaciones, los modelos, acerca de fenómenos o asuntos y sus dimensiones políticas, económicas y sociales (Held, 1993; Tucker, 2001) desarrollados por matrices de pensamiento en áreas o autorías en momentos determinados y frente a realidades, situaciones o coyunturas específicas.

\footnotetext{
${ }^{9}$ Aquí la palabra "categoría" debe ser entendida desde su acepción filosófica y especialmente desde la lógica de Aristóteles y la crítica de Kant. Se refiere por categoría (gr.: Cualidad atribuida a un objeto/fenómeno) a una noción abstracta, un instrumento intelectivo que opera como un insumo de entendimiento con valor ontológico y lógico a través de la detección y clasificación de aspectos como: 1) Cualidad. 2) Cantidad. 3) Relación. 4) Acción. 5) Modalidad. 6) Condición, 7) Sustancia. 8) Situación, entre otras (Aristóteles, 1988; Kant, 2007).
} 


\section{Samuel Pinheiro Guimarães (1939 -)}

En este apartado, se presentará brevemente la biografía profesional y luego la bibliografía intelectual de Samuel P. Guimarães. Aquí, asumiendo al mismo como un intelectual público ${ }^{10}$ que en sus elementos de pensamiento manifiesta la existencia de propósitos con cariz performativo.

\section{Biografía profesional:}

Samuel Pinheiro Guimarães, obtuvo una Licenciatura en Derecho y Ciencias Sociales por la Universidad Federal de Río de Janeiro en Brasil al año 1963. El mismo año, ingresa al Ministerio de Relaciones Exteriores de su país. Dos años más tarde, en 1965, asume la Dirección de la Oficina de Cooperación Internacional para el SUDENE ${ }^{11}$, una agencia gubernamental encargada de alcanzar mayores niveles de desarrollo económico y sostenible en la región nordeste de Brasil a través de la integración competitiva de las bases productivas de la región en la economía nacional e internacional.

Al año 1969, Guimarães obtiene una Maestría en Economía por la Universidad de Boston en Massachussets - Estados Unidos. Por otra parte, desde el año 1995 hasta el año 2001, se desempeñó como director del Instituto para Investigación en Relaciones Internacionales al interior del Ministerio de Relaciones Exteriores de Brasil. Entre enero de 2003 y octubre de 2009, Guimarães ofició como secretario general de la Cancillería brasileña, para luego asumir, hasta el final del Gobierno de Luiz I. Lula da Silva ${ }^{12}$, en diciembre de 2010, el cargo de ministro jefe de la Secretaría de Asuntos Estratégicos de la Presidencia de la República.

Luego de finalizado el gobierno del presidente Lula da Silva, Guimarães asume la posición de alto representante general de Mercosur. Aquí, Guimarães tendrá responsabilidades y funciones vinculadas a articulación política, formulación de propuestas y representación de las posiciones comunes al interior del bloque. Particularmente en esta labor, Guimarães estuvo a cargo de coordinar la aplicación de una serie de objetivos establecidos en un plan de acción para la arquitectura de un Estatuto de Ciudadanía del Mercosur. No obstante, abandona su responsabilidad a través de la renuncia a su puesto al año 2012, acusando falta de apoyo político de Estados miembros de Mercosur (Brasil, Argentina, Uruguay y Paraguay) para la implementación de proyectos. Durante los años 2015 y 2016, Guimarães desarrolló labores de docencia como académico titular en el Instituto Rio Branco de su país ${ }^{13}$ a través de las cátedras "Política Internacional" y "Política Exterior de Brasil".

\footnotetext{
${ }^{10}$ Aquí, se dice intelectual público a una figura que, desde el cultivo de ideas y pensamiento, desarrolla una presencia de guía u orientación significativa en la reflexión de causas societales - públicas (Devés-Valdés, 2019). Para el caso de Guimarães, un ejemplo es la superación del subdesarrollo.

${ }^{11}$ SUDENE: Superintendencia para el Desarrollo del Nordeste. Agencia gubernamental de Brasil cuyo objetivo es impulsar el desarrollo económico en los Estados bajo su jurisdicción.

${ }^{12}$ El Socialista democrático Luiz I. Lula da Silva fue el 35 presidente de Brasil desde 1/1/2003 a 1/1/2011.

${ }^{13}$ Con fundación al año 1945, desde el año 1946 el Instituto Rio Branco (IRBr) es la entidad encargada de la selección y capacitación del personal diplomático de Brasil. Aquí, se lleva a cabo instrucción en las siguientes áreas ligadas a la Diplomacia: 1. Formulación de Política Exterior Nacional, 2) Negociación Internacional, 3) Práctica Consular, 4) Comercio Exterior, 5) Administración Pública, 6) Servicio Exterior, entre otras.
} 
A nuestro modo de ver, la vasta trayectoria y experiencia diplomática es un aspecto que permite a Guimarães el llevar a cabo labores intelectuales asumidas no sólo como tareas descriptivas de la realidad internacional y sus entidades sino, fundamentalmente, como aquellas ligadas a la generación de teorías, modelos y conceptos respecto de procesos empíricos como por ejemplo el crecimiento y desarrollo nacional, la integración institucional hemisférica y las posibilidades de actores de convertirse en potencias globales.

\section{Bibliografía de Guimarães al siglo XXI:}

Al interior del siglo XXI, Samuel P. Guimarães realizará aportaciones al pensamiento latinoamericano respecto asuntos internacionales. Lo anterior, en tanto que aquí se asume a lo que denominamos "pensamiento latinoamericano", como un conjunto compuesto por figuras - obras (producción intelectual), temas (focos o énfasis), problemas (interrogantes, vacíos o desafíos), problemáticas (variables en relación o aleación) y modelos y conceptos dedicados al examen, reflexión y proposición respecto de la región latinoamericana como un todo o desde el tratamiento de alguna de sus partes o aspectos. Aquí, el pensamiento latinoamericano constituye entonces un repertorio de ideas puesto en discusión para entender los fenómenos propios y alcanzar formas de superación (Devés-Valdés, 2000, 2003 y 2019).

Específicamente, las contribuciones esenciales de Guimarães al pensamiento latinoamericano en cuestiones internacionales y mundiales del siglo XXI (Devés-Valdés y Jorquera, 2020) las encontramos en las siguientes obras: Primero, (1999): "500 años de Periferia: Una contribución al Estudio de la Política Mundial". Segundo, (2000): "Visiones Brasileras del Exterior: Argentina, Alemania, África del Sur y Estados Unidos” y tercero, (2005): "Desafíos Brasileños en la Era de los Gigantes".

A continuación, se expondrá un breve resumen de las dos primeras obras de Guimarães y su argumento principal. Luego, se presentarán como a unos de los principales referentes de sentido $^{14}$ para la obra de este autor y su contenido conceptual, a la Geopolítica Clásica de Brasil y a lo que se ha denominado desde la óptica de los Estudios de Pensamiento Latinoamericano como la "Escuela de Brasil Gran Potencia". Aquí, asumiendo a Guimarães como una voz y re - enunciación de esta Escuela a fines del siglo XX y en los primeros años del siglo XXI. Por último, se tratará en detalle el contenido teorético - conceptual de la publicación "Desafíos Brasileños en la Era de los Gigantes" de 2005, entendido el mismo como una síntesis de la propuesta teórica y de conceptos fundamentales de este autor, siempre asumido como un intelectual público y con propósitos o finalidades performativas.

\section{0 años de Periferia. Una contribución al Estudio de la Política Mundial (1999):}

Al interior de esta obra, Guimarães desarrolla un análisis crítico respecto de la reestructuración del orden internacional en el periodo post - guerra fría y sobre las alternativas para que países logren abandonar realidades y condiciones de subdesarrollo y "segunda clase" en el escenario global. Para Guimarães, el orden mundial post - guerra fría se encuentra marcado por una evidente hegemonía de Estados Unidos y, la misma, se caracteriza por el desarrollo significativo de actividades tendientes a la instrumentalización

\footnotetext{
${ }^{14}$ En términos ontológicos y metodológicos - analíticos.
} 
de estructuras de poder nuevas y que inclusive, a juicio de este autor, tendrían la potencialidad para disputar un espacio en la determinación de las formas - posiciones, relaciones de poder y distribución de beneficios - de un emergente sistema mundial. A estas estructuras de poder nuevas, Guimarães refiere específicamente con el nombre de "Grandes Estados Periféricos".

Por Grandes Estados Periféricos, Guimarães define a entidades con las siguientes características: Primero, frágil política y economía. Segundo, estrechos vínculos con países que operan en el sistema internacional, ligados estructuralmente en su evolución, con las dinámicas constituidas por un actor central hegemónico. Tercero, una posición marginal en el escenario global que tiende a agudizarse, a recrudecerse si estos Estados no cumplen con desafíos de comportamiento impuestos por el orden construido desde el Estado hegemónico, a saber, posibilitar la concentración del poder económico, político, militar y cultural del actor central (Guimarães, 1999: 15 - 23).

Guimarães menciona como aspectos específicos de los Grandes Estados Periféricos, a las siguientes aristas: 1) Gran número de población. 2). Gran territorio no inhóspito y razonablemente, potencialmente explotable. 3) Existencia de niveles de desarrollo económico como sostén a la presencia de infraestructuras industriales y a la articulación de mercados internos capaces de asegurar magnitudes mínimas de operación económica y acumulación de capital a escala nacional e internacional. 4) Niveles importantes de subdesarrollo y desigualdades internas que posibilitan una afección positiva y/o negativa de estos países por las ideas, costumbres y políticas generadas por la nación o actor central (Guimarães, 1999: 21 - 23). Al año 1999, este autor refiere como ejemplos de Grandes Estados Periféricos a China, India, Indonesia, Angola, Congo, Mozambique, Sudáfrica, Irán, Brasil, Argentina, Indonesia y México. Todos los países mentados pertenecen en aquel instante al tercer mundo o a áreas en subdesarrollo.

Además de la noción Grandes Estados Periféricos, Guimarães en esta obra introduce el concepto de "Estructura Hegemónica de Poder" o "Histórica Macro - Estructura Hegemónica del Poder" (Guimarães, 1999: 15). Para Guimarães, el sistema internacional se define por esta entidad y la actuación de los Grandes Estados Periféricos ocurre sobre el escenario articulado por la Histórica Macro - Estructura Hegemónica del Poder. En torno a lo expuesto, la entidad aquí referida poseerá las siguientes características:

1) La estructura es histórica porque es capaz de sostener a lo largo de periodos de tiempo significativos, a predominantes actividades específicas, instituciones y a relaciones de poder. 2) El centro o núcleo estructural lo constituyen la unipolaridad militar de Estados Unidos y una realidad de múltiples actores económicamente competitivos. 3) El dominio político de la estructura lo ejerce un Estado hegemónico o central en tanto que, dotado de intereses políticos, militares, económicos y sociales en todas las regiones del mundo, a través de una estrategia particular de perpetuación del orden a su vez definida por criterios como los siguientes: A) Utilización de las Organizaciones Internacionales ${ }^{15}$ como un espacio de cooptación de actores y de difusión de una apariencia de neutralidad ideológica en torno al supuesto respeto de la libre y democrática toma de decisión a nivel internacional por parte de

${ }^{15}$ Ejemplo: Fondo Monetario Internacional (FMI), Banco Mundial (BM) y la Organización Mundial de Comercio (Guimarães, 1999: 35). 
actores. B) Empleo de las Organizaciones Internacionales e instituciones económicas globales para la promoción de una multipolar competencia económica funcional solamente a la política económica de la nación centro. En suma, el papel de Organismos Mundiales se define en tareas de elaboración ideológica y en la generación de diseños técnicos de órganos estatales, políticas y funciones gubernamentales específicas obsecuentes al interés del actor central (Guimarães, 1999: 15 - 35).

Por último, Guimarães en esta obra, luego de describir a la política mundial a través de la especificación de actores y su escenario, lo que hace es adoptar, a nuestro juicio, una posición de intelectual público. Aquí, en tanto que asumir como una "causa propia" al elaborar conocimiento y criterios de movimiento o actuación para que Estados y naciones abandonen su condición histórica de entidades periféricas. De esta manera, Guimarães considerará que una superación de la realidad periférica puede estar dada por el realizar acciones que afirmen los intereses nacionales frente al mundo y esto, a partir de ejercicios como los siguientes:

Primero, potenciar el multilateralismo sur - sur entre América del Sur, África y Asia. Segundo, acometer acciones claras frente a sucesos internacionales a partir del desarrollo de políticas exteriores autónomas. Tercero, priorizar el desarrollo de capacidades nacionales de negociación con potencias desde el abandono de lazos estructurales ${ }^{16}$ de dependencia con los países centro. Cuarto, impulsar procesos de construcción de un mundo multipolar, al alero del desarrollo de alianzas entre países que impliquen una desconcentración de poder en los países centro y un fortalecimiento de la inserción internacional de otros países en el escenario global desde potencialidades políticas, económicas y estratégicas (Guimarães, 1999: 135 141). A través de lo anterior, el propósito o al menos el cariz performativo de la obra de Guimarães queda de manifiesto al intentar "hacer cosas con palabras". Aquí, en tanto que fundamentar guías para el comportamiento mundial de actores en un discurso disciplinariamente articulado, políticamente intencionado y cuyo diagnóstico y propuestas se basan en el empleo de conceptos específicos. A saber, Grandes Estados Periféricos e Histórica Macro - Estructura Hegemónica del Poder.

\section{Visiones Brasileñas del Exterior. Argentina, Alemania, África del Sur y Estados Unidos (2000):}

Al interior de esta obra en serie, compuesta por cuatro publicaciones independientes, Guimarães tratará, con múltiples enfoques y en un marco temporal que va desde 1940 hasta los años 1990 y 2000, a los siguientes ejes vinculados a la política internacional de Brasil: A) Política Exterior de los países seleccionados. B) Tratamiento de la visión que los países seleccionados tienen sobre la política global e internacional. C) Examen de la política exterior - económica de los países escogidos. D) Análisis de los procesos de vinculación e internacionalización llevados a cabo por Brasil con los países seleccionados, siempre considerando como un elemento subyacente a la realidad de globalización (Guimarães, 2000).

En esta serie de publicaciones Guimarães nuevamente, a nuestro juicio, manifiesta un carácter de intelectual público al tener como objetivos centrales a los siguientes dos

\footnotetext{
${ }^{16}$ Políticos, económicos, culturales, científicos, otros.
} 
elementos: Primero, aumentar el conocimiento en Brasil sobre las relaciones internacionales desplegadas con países importantes. Segundo, someter a análisis crítico (develando potencialidades y debilidades) a los cursos de acción acometidos por Brasil en materia de política exterior al interior de la realidad de globalización (Guimarães, 2000).

Hasta aquí, principalmente se han entregado elementos respecto a las dos aportaciones conceptuales elaboradas por Guimarães. No obstante, el despejar algunas interrogantes resulta crucial ¿Qué repertorio epistemológico y conceptual existe antes de la obra de Guimarães?, ¿En qué lugar intelectual, al interior de una tradición de pensamiento regional mayor o histórica, es posible situar a su contenido teorético dadas sus características?

\section{Antes de Guimarães. Análisis geopolítico en Brasil y la Escuela de Brasil Gran Potencia como referentes de sentido:}

A nuestro juicio, tanto el concepto de Grandes Estados Periféricos como el de Histórica Macro - Estructura Hegemónica del Poder constituyen, en la obra de Guimarães, un ejercicio de re - enunciación o segunda enunciación de consideraciones previas elaboradas por autorías ligadas al análisis geopolítico brasileño clásico y a lo que ha sido nombrado como la "Escuela de Brasil Gran Potencia" vigente al menos desde la segunda mitad del siglo XX" (Devés-Valdés, 2020). Lo anterior, se sostiene por las siguientes razones:

En primer lugar, porque los dos conceptos referidos articulan un instrumental esencialmente geopolítico. Aquí, en tanto que consideran de un modo orgánico, como variables determinantes o influyentes sobre la política nacional, internacional y la política exterior, a las características geográficas, a las fuerzas socioculturales y a los recursos económicos ${ }^{18}$. Guimarães es un diplomático profesional. En consecuencia, sus términos son un rendimiento de su histórico - formativa, epistemológica, metodológica y analítica sensibilidad, desde el prisma de los ejercicios de gobierno, a las cuestiones geopolíticas de construcción de soberanía y envergadura o desarrollo estatal en escalas nacionales, regionales y mundiales. Los dos conceptos desarrollados por Guimarães, evidencian su plena conciencia respecto que un Estado soberano, en múltiples niveles, sólo es capaz de alcanzar mayores grados de desarrollo si opera de modo diestro, eficaz y eficiente tanto con características físicas únicas, así como con las formas viables de organización económica, social, política y militar, influenciadas por aquellas características físicas basales. Además, los conceptos expuestos por Guimarães, manifiestan su naturaleza geopolítica pues en colaboración posibilitan, a través de referir a formas específicas de actores y escenarios globales, una comprensión mayor de las relaciones internacionales y mundiales en las que participan Estados y agentes no - estatales.

\footnotetext{
${ }^{17}$ Este texto prioriza la exposición de elementos eidéticos con origen Latinoamericano. No obstante, es posible detectar en la obra de Guimarães referentes desde la Historia, Economía Política Internacional, Derecho Internacional, Ciencia Política y Relaciones Internacionales con origen en EUA y Europa. Ej. G. Arrighi, M. Friedman, K. Galbraith, R. Gilpin, E. Hobsbawm, H. Kelsen, P. Kennedy, W. McNeill, G. Myrdall, P. Renouvin, J. B. Duroselle, J. A. Schumpeter, F. Schurmann, L. Thurrow y J. Williamson entre otros. (Guimarães, 1999 y 2005)

18 Aquí, se suscribe a la visión elaborada por el politólogo Rudolf Kjellén (1864 - 1922) quien en su obra de 1916 "El Estado como Organismo" entendía a la Geopolítica como un escenario de interacción de fuerzas sociológicas, políticas y físicas.
} 
En segundo lugar, los criterios elaborados por Guimarães y particularmente su concepto "Grandes Estados Periféricos" son de un modo evidente un rendimiento de segunda enunciación, un "decir otra vez" de postulados con origen en el análisis geopolítico brasileño clásico y en lo denominado como Escuela de Brasil Gran Potencia. El análisis geopolítico brasileño clásico, también referido por el General Carlos de Meira Mattos como el análisis de los "predecesores" se desarrolla aproximadamente entre los años 1930 hasta 1970. Aquí, la problemática clave desarrollada por autores como Mario Travassos, Everardo Backheuser y Golbery do Couto e Silva, es el asunto de la emancipación o de la superación de una histórica condición de país bajo opresión o marginación. Desde esta perspectiva, la superación enunciada se basa en el desarrollo de una política nacional y exterior internacional que se define por operar sobre las condiciones y posibilidades geopolíticas nacionales procurando su focalización en la obtención de mayores niveles de poder y fuerza, la unión con otros pueblos periféricos y la cristalización de capacidades de liderazgo y conducción hegemónico - geopolítica a nivel regional - sudamericano (Romero, Peña y González, 2012; Devés-Valdés, 2020).

La problemática central del enfoque anterior, es el núcleo analítico de la Escuela de Brasil Gran Potencia. Lo distintivo de esta modulación en la perspectiva geopolítica que se desarrollará entre 1950 / 1970 y hasta aproximadamente las dos primeras décadas de los años 2000, radica en que una posición brasileña de "Gigante" ya no es asumida como restringida al ámbito regional sudamericano, sino que implica un objetivo de constitución de un país con capacidades de ejecutar influencias y provocar rendimientos a escala global. Siguiendo el análisis de los "predecesores", en la Escuela de Brasil Gran Potencia destacan los siguientes autores ligados al argumento de la conversión de Brasil en potencia mundial: Carlos de Meira Mattos, José Luis Fiori, Alberto Moniz Bandeira, Guilherme Sandoval Góes, Nelson Jobin y Samuel P. Guimarães (Devés-Valdés, 2020).

¿De qué manera los criterios y particularmente el concepto Grandes Estados Periféricos de S. P. Guimarães constituyen una segunda enunciación de consideraciones ya expuestas en el abanico del análisis geopolítico brasileño? Particularmente al tratar la cuestión del agente capaz de construir liderazgo internacional (Devés-Valdés, 2020). Aquí, Grandes Estados Periféricos y sus dimensiones e indicadores, no son más, a nuestro juicio, que una representación casi idéntica del Estado brasileño reflexionado por Golbery do Couto e Silva en sus obras "Planeamiento estratégico" (1955) y "Geopolítica de Brasil" (1967) y donde se refería ya a cuatro variables propias a los Estados periféricos como base a la constitución de influencias y liderazgos internacionales y regionales, a saber: 1) Estado suficientemente articulado al interior; 2) Estado efectivamente integrado; 3) Estado expandido en su extenso territorio y 4) Estado con un control manifiesto de sus fronteras (Romero, Peña y González, 2012; Devés-Valdés, 2020). Considerando indicadores constituyentes, el concepto de Guimarães a nuestro modo de ver no puede ser considerado una innovación teorética y sí se presenta como una enunciación lógica derivada de la formación y trayectoria de un diplomático profesional en ejercicio. 


\section{Desafíos Brasileños en la Era de los Gigantes (2005). Refinación conceptual y síntesis teórica:}

En este trabajo, Guimarães expone, a nuestro modo de ver, una síntesis de pensamiento latinoamericano internacionalista. Lo expuesto, dado que retoma un tema y problema central (la relación entre sistema internacional, hegemonías y posibilidades de desarrollo en posiciones iniciales periféricas para actores) y luego revisita y reutiliza los dos conceptos ejes enunciados en su trabajo del año 1999 para refinar sobre todo el término Histórica Macro Estructura Hegemónica del Poder y enlazarlos a otros nuevos conceptos o criterios. Aquí, inclusive con finalidades prospectivas o marcadas por un énfasis de tratar las posibles futuras formas de la realidad internacional. A continuación, se expondrán los principales aspectos de esta obra de Pinheiro Guimarães, entendidos como elementos de acervo eidético en tanto que, dotados de potencialidad para permitir el entender, explicar y actuar al interior del escenario internacional contemporáneo y sus problemáticas.

En "Desafíos Brasileños en la Era de los Gigantes", se plantea un ensayo de proyecto país con miras a operar adecuadamente en el siglo XXI. Se trata entonces, de un trabajo intelectual - analítico y gubernamentalmente intencionado en el que se discuten los variados problemas - económicos, sociales y políticos- del Brasil contemporáneo en función de la realidad internacional, su presente y su futuro posible.

\section{Idea central:}

La idea central de esta obra es que Brasil posee todas las condiciones ${ }^{19}$ para convertirse en una Potencia Mundial o si se quiere, en su lenguaje del año 1999, para abandonar la realidad de periferia y una condición de Gran Estado Periférico en el paso a ser una nación por completo desarrollada. A juicio de Guimarães, el desarrollo de Brasil requiere de la superación de cuatro desafíos específicos: 1) Reducir las disparidades sociales. 2) Superar las dependencias y vulnerabilidades externas. 3) Utilizar todos los potenciales país y 4) Fortalecer la Democracia a nivel nacional y regional (Guimarães, 2005).

Para Guimarães, el que Brasil acometa la tarea de superación de los desafíos referidos no es una cuestión menor. Lo anterior, dado que se asume a aquel camino como el sendero adecuado para habitar en un mundo que transita internacionalmente hacia la multipolaridad y el hacerlo abandonando estadios de miseria, subdesarrollo económico y caos institucional. A lo expuesto, Guimarães denomina como el reto de construcción de un país para la Era de los Gigantes. (Guimarães, 2005).

\section{Histórica Macro - Estructura Hegemónica del Poder:}

Guimarães retoma este concepto de su trabajo del año 1999 para refinarlo y especificarlo en el sentido de precisar algunas variables y los modos en que se vinculan el nivel de política internacional (Estado hegemónico, otros Estados Periféricos y Organizaciones Globales) con el nivel de política interna en Brasil (agentes locales y comportamientos estructurales y estructurantes de estos) en una relación bidireccional (Arriba $\rightarrow$ Abajo y Abajo $\rightarrow$ Arriba) a

\footnotetext{
${ }^{19}$ Geográficas, territoriales, de riqueza en recursos naturales y minerales.
} 
su vez por completo funcional al centro mundial. De esta manera, Guimarães sostiene que los caracteres refinados de esta entidad, como un marco complejo de elementos multinivel, son los siguientes:

A. La presencia al interior países de una clase social hegemónica, un núcleo central o "monopólico" que posee el control sobre las instancias y espacios del poder (instituciones sociales y político-gubernamentales) (Guimarães, 2005).

B. La estructura opera en base a una legislación estatal vinculada de modos múltiples a la alta burocracia nacional y sus intereses (Guimarães, 2005).

C. La estructura funciona a nivel nacional siendo obsecuente a grupos enlazados como los grandes terratenientes rurales, grupos industriales - financieros, a los partidos políticos conservadores, asociaciones civiles y a organizaciones religiosas conservadoras (Guimarães, 2005).

D. La estructura en la escala doméstica, desde su grupo controlador o élite económica, civil y gubernamental, opera perpetuando la concentración del poder político y económico a través de la difusión de un marco ideológico especifico articulado en torno a cuatro dimensiones: 1) Superioridad civilizacional. 2) Raza. 3) Mérito y 4) Desarrollo de promesas religiosas y de progreso ${ }^{20}$. Aquí, destacan como agentes difusores los medios de comunicación de masas, el sistema educativo y como aparato consolidador el código estatal - legislativo (Guimarães, 2005).

Para Guimarães, el núcleo controlador monopólico de la Histórica Macro - Estructura Hegemónica del Poder, dirige al país y lo hace desde la presentación de propuestas de desarrollo nacional que giran en torno a solamente dos puntos de vista u opciones estratégicas con enfoques al interior de su seno: A) Punto de vista económico - enfoque liberal versus enfoque de desarrollo-. B) Punto de vista estratégico / político - enfoque liberal versus enfoque reformista. Aquí, el grupo controlador de la Histórica Macro - Estructura Hegemónica del Poder posibilita una "alternancia" en los espacios de autoridad y una relativa confrontación entre rutas nacionales de desarrollo, pero siempre siendo obsecuentes al centro internacional, a saber, Estados Unidos y al neoliberalismo global. A lo anterior, debe agregarse que nunca se permite la real participación de las capas medias y bajas (capas periféricas) del sistema social en Brasil en el centro legal del país (Guimarães, 2005).

\section{Desarrollo nacional:}

Para Guimarães, el desarrollo nacional debe ser entendido como un conjunto de planes de acción con foco en el tratamiento del empleo y en el abandonar políticas neoliberales que no alientan la economía del país. Aquí, Guimarães sustenta su consideración en el hecho de que las políticas neoliberales, provenientes de países desarrollados, aplican a sociedades con baja densidad demográfica y altos niveles de calificación en su mano de obra (Guimarães, 2005).

\footnotetext{
${ }^{20}$ El marco ideológico expuesto privilegia o prioriza un estereotipo de civilidad, a saber, masculina, blanca, cristiana y capitalista (Guimarães, 2005).
} 
Guimarães, por el contrario, ve que, en su aplicación a contextos de subdesarrollo con diferente situación socioeconómica, lo que hacen las políticas neoliberales es provocar desastrosos impactos a nivel de las diversas esferas del trabajo, a saber, formal, del sector público, comercio informal y actividades ilegales. Para Guimarães, las propuestas neoliberales basadas en una reducción de la esfera pública, lo que hacen es conducir a una hinchazón de la tercera y cuarta esfera laboral debido a que las mismas se definen por no requerir mayores grados de especialización en la mano de obra. Aquí, en definitiva, lo que se impacta es la capacidad del trabajo para generar mayores niveles de riqueza (Guimarães, 2005).

\section{Acción Estatal:}

Para Guimarães, un país desarrollado se vincula a significativos niveles de acción del Estado. Aquí, la actividad estatal debe estar orientada hacia las siguientes áreas: A) Economía: Desarrollo y uso intensivo de los factores productivos a través de su vinculación con políticas nacionales de ciencia y tecnología. B) Desconcentración del poder económico - político: A partir del disminuir la desigualdad de recursos de poder y de facultades consagrada por legislaciones. C) Defensa de los Derechos Humanos: Priorizando la atención sobre grupos históricamente oprimidos ${ }^{21}$ a través del desarrollo de políticas públicas que impliquen la redacción, aplicación y sanción efectiva de normas legales y procedimientos de asignación de recursos (Guimarães, 2005).

\section{Política cultural:}

De acuerdo a Guimarães, una política cultural conducente a mayores niveles de desarrollo país se define por cuestiones como las siguientes: Primero, promoción de la cultura nacional desde un especial aprecio a los atributos con origen en los sectores populares. Segundo, defensa significativa de la pluralidad cultural a través de la promoción de la tolerancia y el aprecio por las formas diversas de nacionalidad (Guimarães, 2005).

En la obra Guimarães la política de cultura constituye, en suma, un dispositivo de "combate" contra la hegemonía cultural externa de países como Estados Unidos y que opera desde medios de comunicación masivos y a partir de una industria cultural ligada al objetivo de perpetuar al interior de países, una conciencia nacional colonizada o, si se quiere, que se define por entenderse a sí misma siempre como inferior. Aquí, se trata de asumir la relevancia de elementos culturales nacionales en función de la construcción de un sentido país y de la proyección de este sentido a nivel global como un activo nacional (Guimarães, 2005).

\section{Política tecnológica:}

Para Guimarães, la política tecnológica constituye un artefacto para lograr mayores niveles de desarrollo económico desde el permitir alientos a las actividades de producción y desde una consecuente expansión del mercado interno en países. Lo anterior, se contrapone con el enfoque neoliberal basado en la apertura de y hacia los mercados, la limitación del alcance del Estado y la adopción del criterio de ventajas comparativas. Este último aspecto, es

\footnotetext{
${ }^{21}$ Personas de color, homosexuales, mujeres, niños y pobres.
} 
entendido por Guimarães como un potenciador de las vulnerabilidades externas de países (Guimarães, 2005).

En la política tecnológica reflexionada por Guimarães, el sector tecnológico es asumido como la materia prima del desarrollo a través su capacidad para generar grandes cantidades de capital a partir del desarrollo de patentes que entregan a los países ventajas competitivas en el mercado de las innovaciones tecnológicas como fuentes de ganancias económicas (Guimarães, 2005).

Guimarães visibiliza, al interior de la desregulación y apertura promovidas por el neoliberalismo, a una ampliación de las fragilidades competitivas de las industrias nacionales. Aquí, dado que a su juicio no existen de hecho, procesos de transferencia tecnológica Norte $\rightarrow$ Sur o Centro $\rightarrow$ Periferia que generen capacidades de competitividad real para las naciones del Sur (Guimarães, 2005).

En definitiva, una adecuada política tecnológica nacional requiere, además, altos niveles de inversión e incentivos a la educación de pregrado y postgrado, con la finalidad de evitar las situaciones de fuga de cerebros y potenciar la creación nacional de avances tecnológicos (Guimarães, 2005).

\section{Superación de la política económica neoliberal:}

¿Cómo es posible articular una ruta alternativa al neoliberalismo económico? De acuerdo a Guimarães, a través del operar bajo los siguientes criterios: A) Asumir a la creación y difusión tecnológica como procesos no naturales sino vinculados al desarrollo de políticas públicas y la regulación de sus procesos. Aquí, se trata de procurar la satisfacción de la demanda económico - social nacional versus el responder a intereses de grades corporaciones multinacionales. B) Considerar que la política productiva y tecnológica debe ser el resultado de una labor de formulación nacional más que el rendimiento de un proceso de importación de políticas (Guimarães, 2005).

\section{Vulnerabilidades de seguridad:}

En la obra de Guimarães, un país desarrollado es capaz de tratar adecuadamente sus vulnerabilidades de seguridad. Algunas relevantes en el escenario global presente son las siguientes: A) Problemas territoriales (relaciones vecinales, estado de la base de recursos naturales, amenazas a la soberanía). B) Inestabilidad política, social y económica de naciones vecinas. C) La acción del Crimen Organizado Transnacional y el Narcotráfico. D). Acción de Nuevas Amenazas en América Latina como el Terrorismo y Fundamentalismos (Guimarães, 2005).

Para Guimarães, un adecuado tratamiento de las vulnerabilidades de seguridad requiere el refuerzo sobre las Fuerzas Armadas Nacionales y sus capacidades de defensa sobre la soberanía y el territorio. Aquí, a partir de mayores grados de inversión en tecnología, armamento y competencias habilitadoras para enfrentar nuevas formas de riesgo y conflictos (Guimarães, 2005). 


\section{Transformaciones del sistema internacional. Siglo XXI y futuro:}

De acuerdo a Guimarães, el sistema internacional durante el siglo XXI puede sufrir significativas transformaciones. Lo expuesto, dado las interacciones posibles entre: A) Número creciente de potencias (Japón, China, Rusia y la Unión Europea). B) Una hiper potencia: Estados Unidos y C) Un conjunto de naciones periféricas en condición de vulnerabilidad (Guimarães, 2005).

Para Guimarães, aquí de lo que se trata, es de que naciones asuman el desafío de conversión en polos de poder o "Gigantes" a partir de articulaciones o alianzas particulares que doten a países de mayores capacidades de actuación y proyección internacional - global. A modo de ejemplo, Guimarães propone para la región sudamericana la conformación de un polo de poder a través del enlace o la integración entre Brasil y Argentina. De acuerdo a Guimarães, la conformación de un polo de poder regional a través de mecanismos de integración multilateral, le permitiría a sur - américa el alcanzar mayores capacidades políticas, económicas y político - exteriores (Guimarães, 2005).

En las líneas previas se han presentado variados elementos conceptuales desarrollados en la obra de Samuel Pinheiro Guimarães en el periodo 1999/2000 a 2005. A nuestro juicio, los mismos poseen, constituyendo un acervo eidético latinoamericano en asuntos internacionales, la potencialidad para reforzar o enriquecer a los Estudios Internacionales en sentido básico y aplicado.

\section{Enriquecimiento básico y aplicado de los Estudios Internacionales desde la obra de Samuel P. Guimarães}

¿Cómo la contribución de Pinheiro Guimarães al acervo eidético internacionalista implica enriquecimientos posibles para la multidisciplina de los Estudios Internacionales en un sentido básico y aplicado? A nuestro juicio, lo anterior se posibilita dado que los elementos de pensamiento generados por Pinheiro Guimarães, pueden ser insumos al alero de dos tareas específicas: 1) Contribuir a la Teoría estándar de Relaciones Internacionales a través de nutrir el Neo - Realismo y 2) Constituir piezas en procedimientos científico - sociales de elaboración de modelos.

\section{Contribución al Neo - Realismo. La obra de Guimarães como ejemplo empírico - analítico de Estructura:}

Kenneth Waltz en su Libro: "Theory of International Politics" de 1979, plantea que el Neorrealismo o Realismo Estructural desarrolla explicaciones de los acontecimientos internacionales fijándose en la Distribución del Poder entre actores o participantes de las relaciones internacionales. Aquí, contrario a una exclusiva concentración sobre el poderío militar de naciones. Waltz, en esta obra, define lo que denomina "Estructura de Poder del Sistema Total de Estados". La "Estructura", es un conjunto jerárquico de relaciones e instituciones, resultante de la definición realizada por los Estados y por la Distribución de Recursos de Poder Efectivo (Uso efectivo) y Poder Potencial (Anuncio, Amenaza, Uso Probable) también realizada por los Estados. Desde lo anterior, la Estructura determina un orden, condicionamiento a relaciones entre Estados como agentes fundamentales y sobre 
otros actores vinculados. En esta propuesta, La Estructura varía, se modifica, con los cambios en la Distribución de Capacidades (Ejercicio de Poder Efectivo y Potencial) entre las Unidades de la Estructura (Estados, relaciones jerárquicas con otros Estados y otras unidades vinculadas a los Estados) y las Capacidades de los Actores implican unidades funcionalmente diferenciadas en su interior con mayor o menor capacidad para cumplir la función básica de las relaciones internacionales para los Neorrealistas, a saber, el asegurar la seguridad.

A nuestro juicio, tanto Grandes Estados Periféricos como Macro - Histórica Estructura Hegemónica de Poder, constituyen una precisión o especificación empírico - analítica de los elementos clave de este enfoque, a saber, Agentes y Estructura. Guimarães, en definitiva, aporta una exposición particular sobre el cómo una estructura sistémica interestatal limita o constriñe el comportamiento de los Estados forzándolos a actuar de manera particular. Sobre todo, la refinación de Guimarães de su concepto de Estructura, implica una modalidad detallada de operación empírica de los elementos teóricos. En Guimarães, los Estados como actores fundamentales, reconociendo además otros nuevos actores (Ej. Organizaciones Internacionales y/o Trans-Nacionales) y Actores Atípicos (Ej. Terrorismo o Narcotráfico) conforman un entramado, un conjunto precisado, controlado de elementos que desarrollan relaciones trans-escalares (de adentro hacia afuera y desde afuera hacia adentro), que buscan acuerdos regionales y supranacionales, a la vez que paralelamente dotan a los Estados mismos, desde su distribución de poder, de una jerarquía, relaciones e instituciones (Ej. Acuerdos formales o alineaciones informales) nuevas que a su vez constriñen su comportamiento.

\section{Contribución en sentido aplicado. Procedimientos de desarrollo de modelos:}

Los conceptos elaborados por Guimarães en conjunto con sus criterios o definiciones prescriptivas, pueden participar en procesos de elaboración modelar. Por modelo, aquí se refiere a la construcción teórica diseñada para revelar y explicar los elementos clave de una forma, fenómeno o proceso y la estructura o las relaciones que le subyacen. Un aspecto de la vida o un conjunto de instituciones sólo pueden ser adecuadamente entendidos en términos de su relación con otros fenómenos sociales. Aquí, Los modelos son, por consiguiente, redes complejas de conceptos y generalizaciones acerca de los aspectos políticos, económicos y sociales (Held, 1993: 21). Estas categorías heurísticas, como redes complejas de conceptos, al ser constituidas por los insumos de Guimarães pueden refinarse en su constante contraste o control empírico y de esta manera, alimentar al pensamiento latinoamericano internacionalista. Los modelos orientados por Guimarães son tantos como las posibles combinatorias de sus elementos constituyentes y cada uno, como ejercicios de elaboración, composición consciente y/o programada de ideas con cariz explicativo, pueden ser una herramienta para la comprensión de la naturaleza de las relaciones internacionales pasadas, presentes y futuras, así como como para el diseño, implementación y evaluación de políticas y políticas públicas. 


\section{Conclusión}

Este escrito ha expuesto la contribución teorética de Samuel P. Guimarães a los Estudios Internacionales en el periodo 1999/2000 - 2005 utilizando como mecanismo de rastreo a la categoría "acervo eidético" proveniente de los Estudios Eidéticos. La contribución mentada puede estilizarse en los conceptos "Grandes Estados Periféricos" e "Histórica Macro Estructura Hegemónica de Poder". Ambos términos, refinados en su vinculación con otros conceptos o criterios como "Desarrollo nacional" y "Acción del Estado", configuran a nuestro modo de ver un esquema o modelo, una pequeña síntesis teórica útil para describir, explicar y prescribir respecto al comportamiento pasado, presente y futuro de actores en el escenario de las relaciones internacionales.

A nuestro juicio, la contribución de Guimarães, puede nutrir las tareas de producción de conocimiento de los Estudios Internacionales en un sentido básico y aplicado a partir de dos dimensiones particulares, por un lado, a través de alimentar teóricamente y empírico analíticamente a la Teoría Neo - Realista de Relaciones Internacionales y a su concepto de "Estructura" y por otro lado, al funcionar como un insumo en procesos científico - sociales de elaboración y contraste de modelos explicativos.

¿De qué otra forma puede resultar útil esta contribución a los Estudios Internacionales y al acervo de pensamiento latinoamericano internacionalista? Específicamente, a través del considerar a la obra de Guimarães en investigaciones bajo la óptica de la Teoría "Pensamiento Periférico" desarrollada por Eduardo Devés-Valdés. Aquí, un ejemplo de lo anterior, radicaría en vincular analíticamente a los conceptos de Guimarães con la "Disyuntiva Periférica" expuesta por Devés-Valdés en su obra y el hacerlo asumiendo a los conceptos y criterios como un rendimiento de una posición intelectual y reflexiva "Identitaria" en contraposición a una postura de actores "Centralitarios" (Devés-Valdés, 2012).

Por último, se considera igualmente que la obra de Guimarães es útil para llevar a cabo discusiones analíticamente provechosas con la Teoría "Realismo Periférico" construida por Carlos Escudé 22 . Aquí, en la medida en que ambas contribuciones se han dotado de una arista práctica al ser aplicadas o al menos, al nutrir cursos de acción al interior de las cancillerías y las políticas exteriores e internacionales de Brasil y Argentina. En suma, se asume que es posible llevar a cabo estudios comparados de los repertorios y extraer conclusiones ligadas tanto a su contenido eidético - teórico como a sus resultados en cuanto ejes de cometidos político exteriores e internacionales.

\footnotetext{
${ }^{22}$ Para indagar sobre la Formulación de Escudé véase: Escudé, C. (1992). “Realismo Periférico”. Buenos Aires: Planeta.
} 


\section{Referencias}

Aristóteles. (1988). "Tratados de Lógica (Órganon). Categorías, tópicos, sobre las refutaciones sofísticas". Madrid: Gredos.

Austin, J L. (2008). “Cómo hacer cosas con palabras”. Barcelona: Paidós.

Auyero, J. (2007). "La zona gris: Violencia colectiva y política partidaria en la Argentina contemporánea”. Buenos Aires: Siglo XXI.

Bello, D. (2013) (Edit.). "Manual de Relaciones Internacionales: herramientas para la comprensión de la disciplina". Santiago: RIL.

Caro, I.; Rodríguez, I. (2013). "El estudio de las Relaciones Internacionales". En: D. Bello (Edit.). Manual de Relaciones Internacionales: herramientas para la comprensión de la disciplina”. Santiago: RIL. PP: $11-32$.

Colombi, B. (2006). "El viaje y su relato". Latinoamérica. (43). PP: 11 - 35.

Devés-Valdés, E. (2000). "El Pensamiento Latinoamericano en el siglo XX. Entre la modernización y la identidad, Tomo 1, del Ariel de Rodó a la CEPAL (1900 - 1950)". 1 ed. Buenos Aires: Biblos - Centro de Investigaciones Diego Barros Arana.

----. (2003). "El pensamiento latinoamericano en el siglo XX. Tomo 2. Desde la CEPAL al neoliberalismo (1950-1990)". Buenos Aires: Biblos - DIBAM.

-----. (2012). "Pensamiento Periférico Asia - África - América Latina - Eurasia y algo más. Una tesis interpretativa global. Santiago de Chile: IDEA-USACH / Ariadna.

-----. (2019). "Pensamiento Latinoamericano entre fines del siglo XX y comienzos del siglo XXI: Temas, Conceptos y Problemas Teóricos”. Seminario Específico, Programa Magíster en Estudios Internacionales (MEI), Instituto de Estudios Avanzados (IDEA), Universidad de Santiago de Chile.

-----. (2020). “Escuela de Brasil Gran Potencia”. En: E. Devés-Valdés y S. T. Álvarez (Eds.). Problemáticas Internacionales y mundiales desde el pensamiento latinoamericano: Teorías, Escuelas, Conceptos, Doctrinas, Figuras" Santiago: Ariadna. PP: 41 - 44.

Devés-Valdés, E.; Álvarez T, S. (2020). "Presentación: Problemáticas Internacionales y mundiales desde el pensamiento latinoamericano: Teorías, Escuelas, Conceptos, Doctrinas, Figuras" Santiago: Ariadna. PP: $11-16$.

Devés-Valdés, E.; Jorquera, C. (2020). "Pensamiento latinoamericano em cuestiones Internacionales y mundiales”. En: E. Devés-Valdés y S. T. Álvarez (Eds.). Problemáticas Internacionales y mundiales desde el pensamiento latinoamericano: Teorías, Escuelas, Conceptos, Doctrinas, Figuras" Santiago: Ariadna. PP: $162-165$.

Freeden, M. (2013). “Ideología. Una brevísima introducción”. España: Universidad de Cantabria.

Held, D. (1993). "Modelos de Democracia”. Madrid: Alianza. 
Guimarães, S P. (1999). “Quinhentos Anos de Periferia: Uma contribuição ao estudo da Política Internacional”. Porto Alegre: Ed. Da Universidade Federal do Rio Grande do Sul (UFRGS) - Contraponto.

-----. (Org.). (2000). “África do Sul: Visões Brasileiras”. Brasilia: Instituto de Pesquisa de Relações Internacionais (IPRI) - ITAMARATY.

-----. (Org.). (2000). “Alemanha: Visões Brasileiras”. Brasilia: IPRI.

-----. (Org.). (2000). “Argentina: Visões Brasileiras”. Brasilia: IPRI.

-----. (Org.). (2000). “Estados Unidos: Visões Brasileiras”. Brasilia: IPRI.

-----. (2006). “Desafios brasileiros na era dos gigantes”. Rio de Janeiro: Contraponto.

-----. (2011). “Los caminos de la integración ¿Hacia dónde vamos?” (Internet). Observatorio de Política Exterior, CEPES - Argentina. Disponible en: https://www.youtube.com/watch?v=B_KW4zLaBX4\&t=137s (Acceso: Mayo, 2021).

Heiss, C. (2015). “Ciencia Política en Chile: ¿Una disciplina consolidada?”. Revista de Ciencia Política. Vol. 35, Nro. 1. Pp: $47-70$.

Kant, I. (2007). “Crítica de la razón pura”. Buenos Aires: Colihue.

Keohane, R.; Nye, J. (1989). "Power and Interdependence: World Politics in Transition". Little, Brown and Company.

Mella, M. (2010). "Elementos de Ciencia Política apuntes de clases: Cultura, conceptos y actores políticos”. Santiago: USACH.

----. (2012). "Elementos de Ciencia Política Volumen 1: Conceptos, actores y procesos". Santiago: RIL - USACH.

Muñoz, H. (1980). "Los Estudios Internacionales en América Latina: problemas fundamentales”. Estudios Internacionales, Vol. 13, No. 51. PP: 328 - 344.

Navarrete, B.; Morales, M.; Figueroa, P. (2005). "La Ciencia Política en Chile y el estado de su docencia". Estudios Sociales. 116, 2. PP: 25 - 52.

Orrego V, F. (1981). "Los Estudios Internacionales en América Latina: realizaciones y desafíos". Santiago: Instituto de Estudios Internacionales de la Universidad de Chile.

Rodríguez, I. (2013). “Teoría de las Relaciones Internacionales: del Primer al Tercer Debate”. En: D. Bello (Edit.). Manual de Relaciones Internacionales: herramientas para la comprensión de la disciplina”. Santiago: RIL. PP: 85 - 126.

Romero, M.; Peña G, R.; González, P. (2012). "Brasil: raíces geopolíticas y actual influencia en expansión”. Política y cultura, (37). PP: 233 - 253.

Ross, C. (2020). “Área de Estudios Internacionales: Experiencia, logros y desafíos”. Sextas Jornadas de Estudios Internacionales, Instituto de Estudios Avanzados (IDEA), Universidad de Santiago de Chile. 
Salomón, M. (2013). "Nuevas corrientes en la teoría de las Relaciones Internacionales”. En: En: D. Bello (Edit.). Manual de Relaciones Internacionales: herramientas para la comprensión de la disciplina”. Santiago: RIL. PP: 127 - 166.

Touchard, J. (2006). “Historia de las Ideas Políticas”. España: Tecnos.

Tucker, I. (2001). "Survey of Economics”. USA: South-Western College.

Viacava G, J. (2012). "La Ciencia Política en Chile: una carrera en expansión y transformación". Política, Vol. 50, Nro. 1. PP.: 93 - 110.

Waltz, K. (1979). “Theory of International Politics”. New York: Mac-Graw-Hill. 